\title{
Thyroid status and nitric oxide in rat arterial vessels
}

\author{
R M McAllister, I Albarracin, E M Price ${ }^{1}$, T K Smith, J R Turk ${ }^{1}$ \\ and $\mathbf{K} \mathbf{D}$ Wyatt
} Departments of Anatomy and Physiology and Kinesiology, Kansas State University, Manhattan, Kansas 66506, USA
'Department of Veterinary Biomedical Sciences, University of Missouri, Columbia, Missouri 65211, USA
(Requests for offprints should be addressed to R M McAllister, Department of Anatomy and Physiology, Kansas State University, 228 Coles Hall, Manhattan,
Kansas 66506, USA; Email: mcallist@vet.ksu.edu)

\begin{abstract}
Thyroid disease has profound effects on cardiovascular function. Hypo- and hyperthyroidism, for example, are associated with reduced and increased maximal endothelium-dependent vasodilation respectively. We therefore hypothesized that the capacity for vascular nitric oxide (NO) formation is decreased in hypothyroidism and increased in hyperthyroidism. To test this hypothesis, rats were made hypothyroid (HYPO) with propylthiouracil or hyperthyroid (HYPER) with triiodothyronine over 3-4 months. Compared with euthyroid control rats (EUT), HYPO exhibited blunted growth and lower citrate synthase activity in the soleus muscle; HYPER exhibited left ventricular hypertrophy and higher citrate synthase activity in the soleus muscle ( $P<0 \cdot 05$ for all effects). The capacity for $\mathrm{NO}$ formation was determined in aortic extracts by formation of $\left[{ }^{3} \mathrm{H}\right] \mathrm{L}$-citrulline from $\left[{ }^{3} \mathrm{H}\right] \mathrm{L}-$
\end{abstract}

arginine, i.e. NO synthase (NOS) activity. Thyroid status modulated NOS activity (EUT, $36 \cdot 8 \pm 5 \cdot 5 \mathrm{fmol} / \mathrm{h}$ per $\mathrm{mg}$ protein; HYPO, 26.0 $\pm 7 \cdot 9$; HYPER, $64 \cdot 6 \pm 12 \cdot 7$; $P<0 \cdot 05$, HYPER vs HYPO). Expression of endothelial and neural isoforms of NOS was modulated by thyroid status in a parallel fashion. Capacity for responding to $\mathrm{NO}$ was also determined via measuring cGMP concentration in aortae incubated with sodium nitroprusside. Stimulated cGMP formation was also modulated by thyroid status (EUT, $73 \cdot 0 \pm 20 \cdot 2 \mathrm{pmol} / \mathrm{mg}$ protein; HYPO, 152.4 \pm 48.7; HYPER, $10 \cdot 4 \pm 2 \cdot 6 ; P<0 \cdot 05$, HYPER vs HYPO). These data indicate that thyroid status alters capacities for both formation of and responding to NO. The former finding may contribute to previous findings concerning vascular function in thyroid disease states.

Journal of Endocrinology (2005) 185, 111-119

\section{Introduction}

Thyroid disease is estimated to affect $10 \%$ of the population (Tunbridge \& Caldwell 1991, Canaris et al. 2000). A hallmark of two common forms of thyroid disease, hypo- and hyperthyroidism, is exercise intolerance. Several studies have examined various mechanisms potentially responsible for exercise intolerance in these disease states. In hypothyroidism, it appears that cardiovascular dysfunction is largely responsible for poor exercise tolerance; in hyperthyroidism, other factors such as hyperthermia and altered patterns of substrate utilization are implicated (cf. McAllister et al. 1995a).

Cardiovascular dysfunction during exercise in hypothyroidism is profound. We have previously reported that skeletal muscle blood flow during treadmill running is reduced $\sim 50 \%$ in hypothyroid rats compared with their euthyroid counterparts (McAllister et al. 1995b). Lower blood flow would impair delivery of $\mathrm{O}_{2}$ and substrates to exercising muscle. When vasomotor responses were determined for euthyroid and hypothyroid rats, endothelium-dependent dilation was also found to be blunted in isolated aortae (Delp et al. 1995, McAllister et al. 2000) and isolated perfused kidneys (Vargas et al. 1995) in the hypothyroid state. Paradoxically, given that exercise tolerance is reduced, hyperthyroidism is associated with enhanced muscle blood flow during exercise (Frey 1967, McAllister et al. 1995c) and augmented endotheliumdependent vasodilation (Scivoletto et al. 1986, Vargas et al. 1995, McAllister et al. 1998, 2000, Honda et al. 2000). Thus, in both thyroid disease states, alterations in endothelium-dependent vasodilation are consistent with patterns of blood flow to exercising skeletal muscle.

Endothelium-independent vasodilation has generally been reported to be normal in both the hypothyroid (Delp et al. 1995, McAllister et al. 2000) and hyperthyroid states (McAllister et al. 1998, 2000), although exceptions have been reported (Vargas et al. 1995, Honda et al. 2000). Collectively, these findings suggest that thyroid status primarily affects the vascular endothelium. Endotheliumdependent dilation of the rat aorta is primarily mediated by nitric oxide (NO) (Delp et al. 1993). We therefore hypothesized that altered endothelium-dependent vasodilation with changes in thyroid status is due to changes 
in the capacity for $\mathrm{NO}$ formation, as reflected by $\mathrm{NO}$ synthase (NOS) activity. We also hypothesized that the response to $\mathrm{NO}$, as reflected by the capacity of vascular smooth muscle to generate cGMP, is not altered by thyroid status.

\section{Materials and Methods}

\section{Animal treatments}

Two series of male Sprague-Dawley rats (Charles River, Wilmington, MA, USA), initially weighing 150-175 g, were housed three per cage in a room with controlled temperature $\left(20-21^{\circ} \mathrm{C}\right)$ and light $(12 \mathrm{~h}$ light: $12 \mathrm{~h}$ darkness). In each series, rats were randomly assigned to one of three groups: euthyroid control (EUT), hypothyroid (HYPO) and hyperthyroid (HYPER). HYPO rats were made hypothyroid over a 3-4 month period with propylthiouracil in their drinking water $(0 \cdot 04 \mathrm{~g} / 100 \mathrm{ml})$, as done previously (Delp et al. 1995, McAllister et al. 1995b, 2000). HYPER rats were made hyperthyroid over 3-4 months with i.p. injections of triiodothyronine $(300 \mu \mathrm{g} / \mathrm{kg}$ in $0.50 \mathrm{mM} \mathrm{NaOH})$ on alternate days, as done previously (McAllister et al. 1995c, 1998, 2000) All rats were allowed free access to food and water except for EUT, which were slightly food-restricted ( $\sim 90 \%$ of normal food intake) over the final month of the treatment period to match body weights with those of HYPO and HYPER. This mild degree of food restriction would not affect thyroid status in EUT, since only either starvation or severe food restriction has been reported to reduce thyroid hormone levels (cf. Danforth \& Burger 1989). All treatments were approved by the Institutional Animal Care and Use Committee of Kansas State University, and were in accordance with the NIH Guide for the Care and Use of Laboratory Animals.

\section{Treatment efficacy}

Efficacy of propylthiouracil and triiodothyronine treatments was assessed by determination of citrate synthase activity in the soleus muscle, gain in body weight during the treatment period and left ventricular weight/body weight ratio. Activity of citrate synthase, a marker enzyme for oxidative capacity, was determined spectrophotometrically (Shimadzu UV-1201, Shimadzu, Kyota, Japan) according to the method of Srere (1969), as done previously to document hypo- and hyperthyroidism (Delp et al. 1995, McAllister et al. 1995b,c, 1998, 2000).

\section{NOS activity}

With the first series of rats (NOS Series), capacity for vascular NO formation was assessed by determining NOS activity in aortic extracts of EUT, HYPO and HYPER. Extracts of aortae were prepared in the following manner: the entire aorta was excised, quickly cleaned of fat and connective tissue under a dissecting microscope (Nikon, Melville, NY, USA), and cut into rings $\sim 1 \mathrm{~mm}$ in axial length. Vascular rings were transferred to microcentrifuge tubes containing $250 \mu \mathrm{l}$ of a buffer solution containing (in $\mathrm{mM}$ unless otherwise indicated): 50 Tris- $\mathrm{HCl}, 0 \cdot 10$ EDTA, $0 \cdot 10$ EGTA, $0 \cdot 10 \mathrm{ml} / 100 \mathrm{ml} \beta$-mercaptoethanol, 20 CHAPS, 1.0 phenylmethylsulfonyl fluoride, 0.002 leupeptin and $0 \cdot 001$ pepstatin $\mathrm{A}, \mathrm{pH} \mathrm{7.8}$. Vascular rings were sonicated (Fisher Sonic Dismembrator 60, Fisher, Pittsburgh, PA, USA) in this buffer solution (5 s, three times), and then centrifuged at $250000 \mathrm{~g}$ (Beckman TL-100 ultracentrifuge, Beckman, Palo Alto, CA, USA) for $30 \mathrm{~min}$ at $4{ }^{\circ} \mathrm{C}$. Aliquots of the resultant supernatant were assayed for NOS activity.

NOS activity was determined according to the method of Pollock et al. (1991), in which the conversion of $\left[{ }^{3} \mathrm{H}\right] \mathrm{L}$-arginine to $\left[{ }^{3} \mathrm{H}\right] \mathrm{L}$-citrulline is measured. Aortic extracts were incubated for $1 \mathrm{~h}$ at $27^{\circ} \mathrm{C}$ in a buffer solution with (final concentration, in $\mathrm{mM}$ unless otherwise indicated): 50 Tris- $\mathrm{HCl}, 54 \mathrm{~L}$-valine, $1.2 \mathrm{MgCl}_{2}, 1 \cdot 0$ $\mathrm{NADPH}, 0.01$ tetrahydrobiopterin, $0.005 \mathrm{~L}$-arginine, 50 $\mathrm{U} / \mathrm{ml}$ calmodulin and $\left.0 \cdot 002 \mathrm{FAD}, \mathrm{pH} 7 \cdot 8 .{ }^{3} \mathrm{H}\right] \mathrm{L}$-arginine (0.010 mCi; New England Nuclear, Boston, MA, USA) was also included. Aliquots of extracts were incubated in this buffer solution that contained $\mathrm{CaCl}_{2}(1.78 \mathrm{mM})$ and in buffer solution that did not contain $\mathrm{CaCl}_{2}$ to determine $\mathrm{Ca}^{2+}$-dependent NOS activity. Appropriate backgrounds (i.e. aliquots of buffer solution rather than aortic extract) were incubated in parallel. At the end of the incubation period, the NOS reaction was arrested with addition of a buffer solution containing $20 \mathrm{mM}$ HEPES and $8.0 \mathrm{mM}$ EDTA, pH 5.5. Reaction mixtures were loaded onto cation exchange columns (Dowex AG 50WX-8, $\mathrm{Na}^{+}$ form; Bio-Rad), and $\left.{ }^{3} \mathrm{H}\right] \mathrm{L}$-citrulline was eluted from columns with $0.50 \mathrm{ml}(\times 2) d d \mathrm{H}_{2} \mathrm{O}$. Scintillation fluid was added to eluent, and samples were counted in a liquid scintillation counter (Packard, Meriden, CT, USA).

\section{NOS expression}

Western blotting was used to determine expression of the endothelial and neural isoforms of NOS (eNOS and nNOS respectively), as well as that of guanylate cyclase (GC), in aortae from EUT, HYPO and HYPER. Aortic extracts prepared for determination of NOS activity (see above) were subsequently used in Western blotting experiments. For each sample (eNOS, nNOS), a volume of extract containing $5.0 \mu \mathrm{g}$ total protein was used. For GC, $2.5 \mu \mathrm{g}$ total protein were used. Samples were loaded onto a $4-12 \%$ Bis-Tris gel, and electrophoresed at $200 \mathrm{~V}$ for $50 \mathrm{~min}$. Transfer to polyvinylidene difluoride membrane was done at $200 \mathrm{~mA}$ for $2 \mathrm{~h}$. Blocking was done for $60 \mathrm{~min}$ in Tris-buffered saline with $0 \cdot 10 \mathrm{ml} / 100 \mathrm{ml}$ Tween 20 and $5 \cdot 0 \mathrm{~g} / 100 \mathrm{ml}$ non-fat milk (TBST-milk). Following blocking, overnight incubation with a primary antibody 
(in TBST-milk) against eNOS (1:1250; BD Transduction Laboratories), nNOS (1:1000; BD Transduction Laboratories), GC (1:5000; Cayman Chemical, Ann Arbor, MI, USA), or glyceraldehyde-3-phosphate dehydrogenase (GAPDH) (1:2000; Chemicon, Temecula, CA, USA) was done. Separate membranes were generated and probed for eNOS, nNOS and GC; all membranes were subsequently re-probed for GAPDH. After washing, blots were incubated for $60 \mathrm{~min}$ with a secondary antibody (horseradish peroxidase-conjugated anti-mouse; 1:2500, in TBST-milk; Sigma-Aldrich), washed, and then visualized on film using enhanced chemiluminescence (Amersham Pharmacia Biotech). Densitometry software (NIH) was used to quantitate protein expression, with eNOS, nNOS and GC expression being normalized to GAPDH expression.

\section{Immunohistochemistry}

As described in detail previously (Henderson et al. 2004), $5 \mu \mathrm{m}$ sections of fixed $(10 \mathrm{ml} / 100 \mathrm{ml}$ formalin), paraffinembedded aortic rings were cut with an automated microtome (Microm, Walldorf, Germany), floated onto positively-charged slides, and de-paraffinized. Sections were incubated with avidin-biotin two-step blocking solution (Vector SP-2001, Vector, Burlingame, CA, USA) to inhibit background staining and with hydrogen peroxide $(3 \mathrm{ml} / 100 \mathrm{ml})$ to inhibit endogenous peroxidase. Non-serum protein block (Dako X909, Dako, Carpinteria, CA, USA) was used to inhibit non-specific binding. Tissue sections were incubated overnight with primary antibodies against eNOS (mouse monoclonal, 1:800; BD Transduction Laboratories, San Diego, CA, USA) and nNOS (rabbit polyclonal, 1:100; Zymed, San Francisco, CA, USA) at $4{ }^{\circ} \mathrm{C}$. After washing, sections were incubated with biotinylated anti-mouse or rabbit secondary antibodies in PBS containing $15 \mathrm{mM}$ sodium azide and peroxidase-labeled streptavidin (Dako LSAB+ kit, peroxidase; K0690). For negative controls, histological sections were prepared as described above, but incubation with primary antibody was excluded from the protocol. Sections were examined and photographed using an Olympus BX40 photomicroscope (Olympus, St. Louis, MO, USA).

\section{cGMP formation}

With the second series of rats (cGMP Series), the concentration of cGMP under basal conditions and upon stimulation with an NO donor was determined in aortae from EUT, HYPO and HYPER. To prepare aortae for cGMP determination, vessels previously excised, cleaned and frozen at $-80{ }^{\circ} \mathrm{C}$ (entire aorta for basal levels, abdominal aorta for stimulated levels) were opened longitudinally, pinned to the bottom of a dissecting dish filled with ice-cold DMEM-HEPES modification buffer solution, $\mathrm{pH} 7 \cdot 4$, and gently denuded of their endothelium with a cotton-tipped applicator. Vessels were then placed in DMEM-HEPES modification buffer solution with $1.0 \mathrm{mM}$ isobutylmethylxanthine, $\mathrm{pH} 7 \cdot 4$ (basal) or the same buffer solution with $10^{-4} \mathrm{M}$ sodium nitroprusside, an $\mathrm{NO}$ donor, also included (stimulated). After a $30 \mathrm{~min}$ incubation period, the GC reaction was arrested with $70 \%$ trichloroacetic acid and then neutralized with $10 \cdot 0 \mathrm{M}$ $\mathrm{KOH}$. Vessels were homogenized (IKA Labortechnik T25 homogenizer, Labortechnik, Staufen, Germany) using $10 \mathrm{~s}$ bursts until complete homogenization was achieved. Homogenates were centrifuged (Damon PR-J centrifuge) at $1500 \mathrm{~g}$ for $10 \mathrm{~min}$. The resultant supernatant was assayed for cGMP concentration; the pellet was used for determination of protein concentration (see below). Concentrations of cGMP in supernatants was determined using an RIA kit (Amersham Pharmacia Biotech).

In addition to determining aortic cGMP concentration, experiments were conducted to determine release of cGMP from aortae. Stimulation of cGMP formation in whole aortae (sectioned into $1 \mathrm{~mm}$ axial length rings) using an NO donor was done as described above. After centrifugation $(1500 \mathrm{~g}$ for $10 \mathrm{~min}$ ) to pellet aortic rings, cGMP concentration in supernatants was determined with an enzyme immunoassay kit (Assay Designs, Ann Arbor, MI, USA).

\section{Protein concentration}

Protein concentration was determined on aortic extracts to normalize NOS activities, and on pellets of homogenized vessels to normalize cGMP concentrations. Protein concentration was determined with a kit utilizing the Bradford procedure (Bio-Rad).

\section{Statistical analysis}

All data are presented as means \pm S.E. Data for EUT, HYPO and HYPER were analyzed using one-way ANOVA (Steel \& Torrie 1980). When ANOVA indicated a difference among groups, the Tukey test was used as a post-hoc test to discern differences between pairs of groups (Steel \& Torrie 1980). $P<0 \cdot 05$ was considered significant for all analyses.

\section{Results}

\section{Treatment efficacy}

Treatment of rats with propylthiouracil successfully induced the hypothyroid state. Citrate synthase activity was lower in HYPO than EUT in the soleus muscle for both series of rats (Fig. 1). In addition, HYPO failed to increase body weight at a normal rate, reflected by lower final body weights than those of EUT and HYPER, in both series (Table 1). Similarly, two lines of evidence indicated that treatment of rats with triiodothyronine induced the 


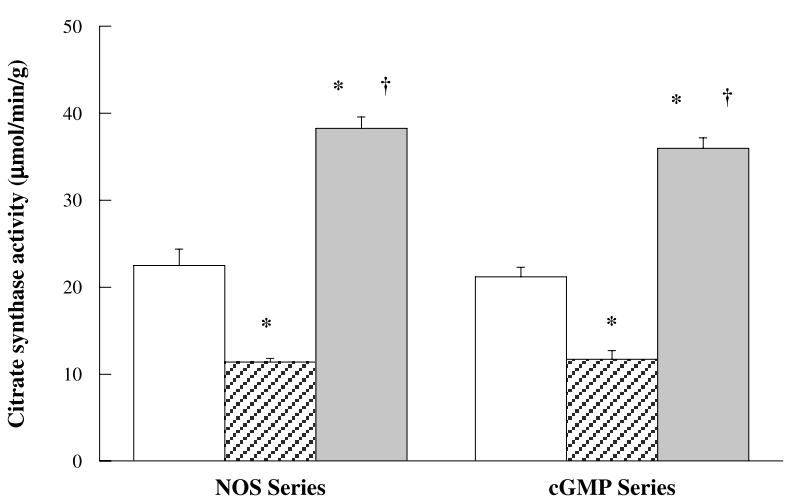

Figure 1 Citrate synthase activity in soleus muscles of rats of the NOS Series and cGMP Series. Values are means \pm S.E.; $n=7,7$ and 8 for Euthyroid (open bars), Hypothyroid (hatched bars) and Hyperthyroid (stippled bars) respectively in the NOS Series; $n=12$, 11 and 10 for Euthyroid, Hypothyroid and Hyperthyroid respectively in the CGMP Series. *Different from Euthyroid, $P<0 \cdot 05 ;$ tdifferent from Hypothyroid, $P<0 \cdot 05$.

Table 1 Indicators of treatment efficacy. Values are means \pm S.E. For NOS Series $n=7,7$ and 8 for Euthyroid, Hypothyroid and Hyperthyroid respectively; for cGMP Series $n=12,10$ and 10 for Euthyroid, Hypothyroid and Hyperthyroid respectively

\begin{tabular}{|c|c|c|}
\hline & NOS Series & cGMP Series \\
\hline \multicolumn{3}{|c|}{$\begin{array}{l}\text { LV weight/ } \\
\text { body weight ratio }(\mathrm{mg} / \mathrm{g})\end{array}$} \\
\hline Euthyroid & $2 \cdot 03 \pm 0 \cdot 08$ & $1.93 \pm 0.03$ \\
\hline Hypothyroid & $1 \cdot 86 \pm 0 \cdot 05$ & $2 \cdot 10 \pm 0 \cdot 06$ \\
\hline Hyperthyroid & $2 \cdot 50 \pm 0 \cdot 10^{* \dagger}$ & $2 \cdot 45 \pm 0.07^{\star \dagger}$ \\
\hline \multicolumn{3}{|c|}{ Final body weight (g) } \\
\hline Euthyroid & $497 \pm 15$ & $516 \pm 15$ \\
\hline Hypothyroid & $284 \pm 6^{*}$ & $253 \pm 6^{*}$ \\
\hline Hyperthyroid & $478 \pm 15^{\dagger}$ & $493 \pm 15^{\dagger}$ \\
\hline
\end{tabular}

LV, left ventricle. *Different from Euthyroid in same series, $P<0 \cdot 05 ;{ }^{\dagger}$ different from Hypothyroid in same series, $P<0 \cdot 05$.

hyperthyroid state. First, citrate synthase activity was increased in HYPER (vs EUT) soleus muscles in both series of rats (Fig. 1). Secondly, left ventricular weight/body weight ratio was increased in HYPER compared with EUT (Table 1), reflecting left ventricular hypertrophy.

\section{NOS activity and expression}

Figure 2 illustrates $\mathrm{Ca}^{2+}$-dependent NOS activity for EUT, HYPO and HYPER aortic extracts. Thyroid status significantly modulated NOS activity, with values for HYPER being more than twice those for HYPO $(P<0 \cdot 05)$. Neither HYPO nor HYPER, however, differed significantly from EUT in NOS activity. In two control experiments, aortae from normal rats demonstrated a mean reduction of $81 \%$ in $\mathrm{Ca}^{2+}$-dependent NOS activity when treated with the competitive NOS inhibitor $\mathrm{N}^{\mathrm{G}}$-monomethyl-L-arginine (4.29 mM) (Pollock et al.

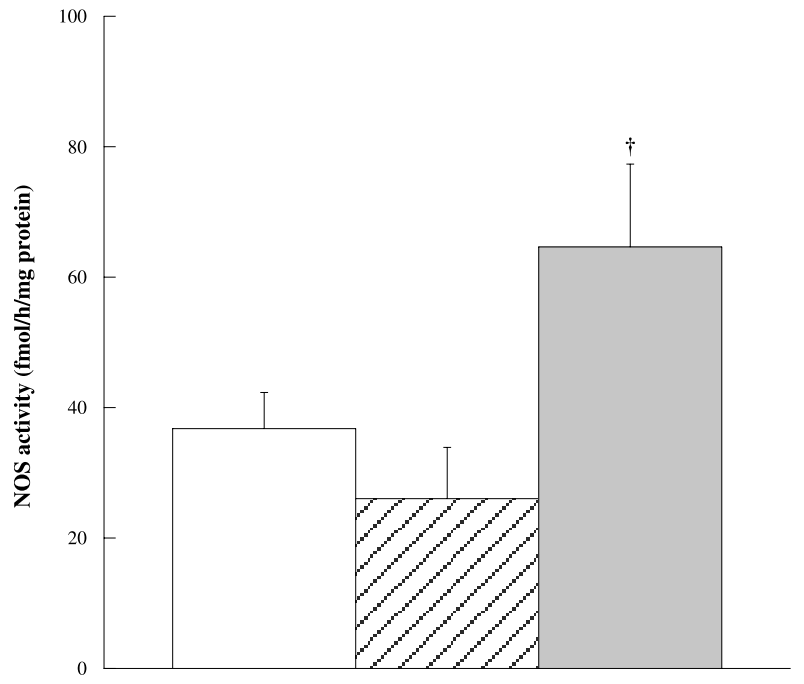

Figure $2 \mathrm{Ca}^{2+}$-dependent NOS activity in aortic extracts. Values are means \pm S.E.; $n=7,7$ and 8 for Euthyroid (open bar), Hypothyroid (hatched bar) and Hyperthyroid (stippled bar) respectively. †Different from Hypothyroid, $P<0 \cdot 05$.

1991). eNOS and nNOS protein expression differed significantly between HYPER and HYPO in a parallel fashion, as illustrated in Fig. 3. Immunohistochemistry for eNOS (Fig. 4) and nNOS (Fig. 5) demonstrated markedly greater nNOS expression in vascular smooth muscle of HYPER (Fig. 5). Expression of eNOS was confined to endothelium in all groups and did not differ visibly among groups (Fig. 4).

\section{cGMP concentration}

Basal levels of cGMP were not different among groups, being $0 \cdot 214 \pm 0 \cdot 055, \quad 0 \cdot 181 \pm 0 \cdot 051$ and $0 \cdot 101 \pm$ $0.027 \mathrm{pmol} / \mathrm{mg}$ protein for EUT $(n=4)$, HYPO $(n=3)$ and HYPER $(n=4)$ respectively. Figure 6A illustrates data for stimulated levels of cGMP. Stimulation of GC with sodium nitroprusside increased cGMP concentration substantially over basal levels for EUT, HYPO and HYPER. Stimulated cGMP concentration attained was, similar to NOS activity, significantly modulated by thyroid status. Values for HYPO were significantly greater than those for HYPER $(P<0 \cdot 05)$; however, neither HYPO nor HYPER were significantly different from EUT. GC protein expression did not differ significantly among EUT, HYPO and HYPER (Fig. 6B).

Thyroid status can affect tissue protein concentration; thus, data for cGMP levels were also expressed and analyzed on a per tissue weight basis. Thyroid status did not affect basal cGMP concentration (EUT, 7.2 $\pm 1 \cdot 0 \mathrm{pmol} / \mathrm{g}$; HYPO, $12 \cdot 7 \pm 3 \cdot 2$; HYPER, $6 \cdot 2 \pm 1 \cdot 5$; ns). Stimulated cGMP concentration was, however, modulated by thyroid state (EUT, 463.8 $\pm 110 \cdot 1 \mathrm{pmol} / \mathrm{g}$; HYPO, 334.5 $\pm 65 \cdot 6$; HYPER, $82 \cdot 2 \pm 8 \cdot 8 ; P<0 \cdot 05$, HYPER vs EUT, HYPO). 

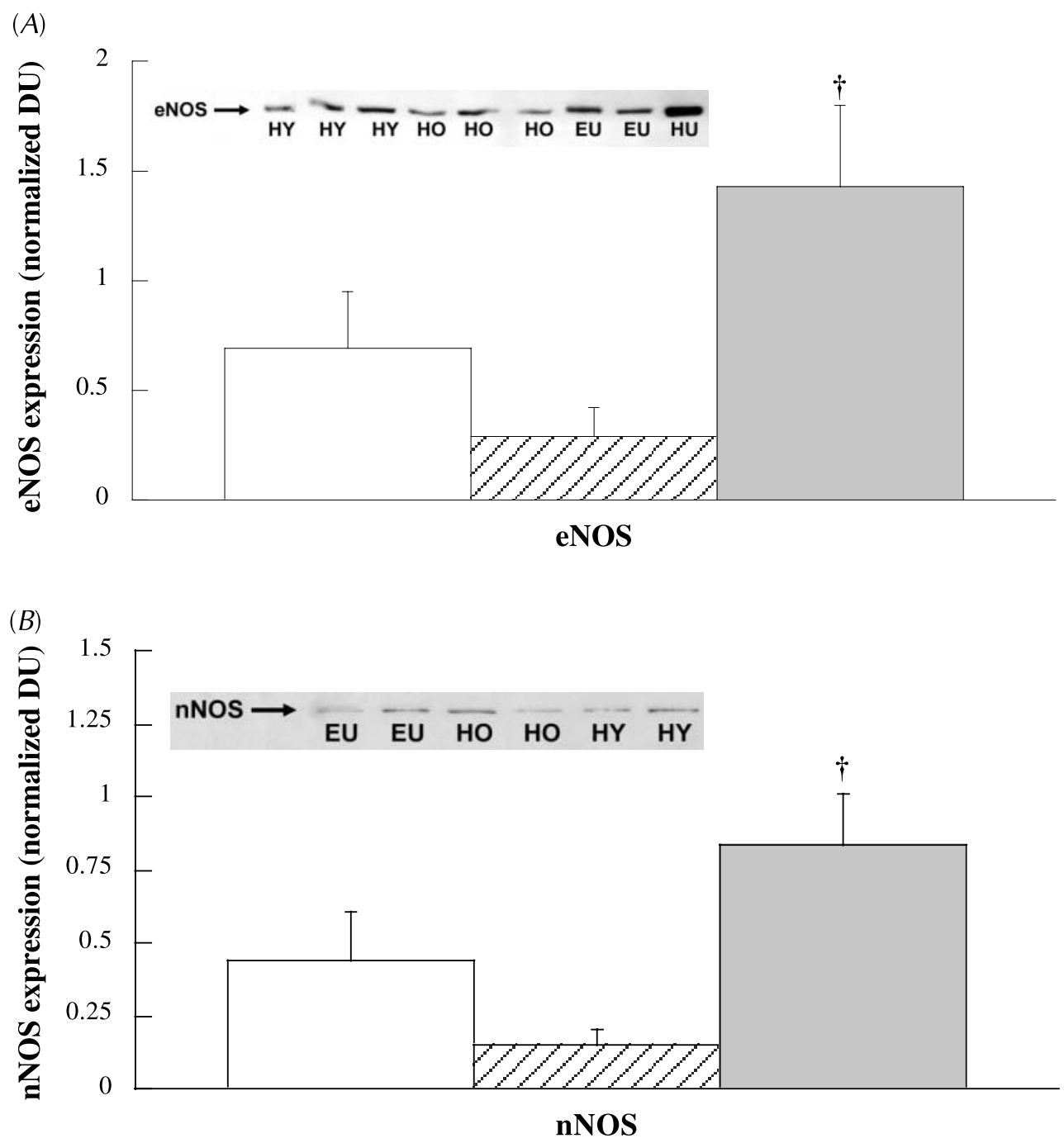

Figure 3 Expression of eNOS (A) and nNOS (B). Values are means \pm s.E.; $n=7,6$ and 8 for Euthyroid (open bars), Hypothyroid (hatched bars) and Hyperthyroid (stippled bars) respectively for eNOS; $n=6,6$ and 8 for Euthyroid, Hypothyroid and Hyperthyroid respectively for nNOS. DU, densitometry units, normalized to GAPDH. †Different from Hypothyroid, $P<0 \cdot 05$. Insets are representative Western blots for eNOS and nNOS. EU, Euthyroid; HO, Hypothyroid; HY, Hyperthyroid; HU, human umbilical vein endothelial cell extract.

When release of cGMP from vascular smooth muscle was determined, cGMP concentration in the incubation medium was also elevated in HYPO (EUT, 0.057 \pm $0 \cdot 11 \mathrm{pmol} / \mathrm{ml}$ per $\mathrm{mg}$ wet weight, $n=7$; HYPO, $0 \cdot 110 \pm 0 \cdot 017, \quad n=9 ; \quad$ HYPER, $0 \cdot 072 \pm 0 \cdot 010, \quad n=8 ;$ $P<0 \cdot 05$, HYPO vs EUT).

\section{Discussion}

The key findings of this study are that capacities for both formation of and responding to NO in a rat arterial vessel are modulated by thyroid status. The hyperthyroid state was associated with increased capacity for NO formation, but reduced capacity for responding to NO, compared with the hypothyroid state. Capacity for NO formation, assessed by NOS activity, is primarily a property of the vascular endothelium. Capacity for responding to NO, estimated by $\mathrm{NO}$ donor-stimulated concentration of cGMP, resides in vascular smooth muscle. These data indicate that thyroid status affects more than the endothelial layer of the vascular wall, contrary to what a majority of previous studies had suggested.

\section{Rat models of hypo- and hyperthyroidism}

Treatment of rats with propylthiouracil was successful in inducing the hypothyroid state. Citrate synthase activity, 


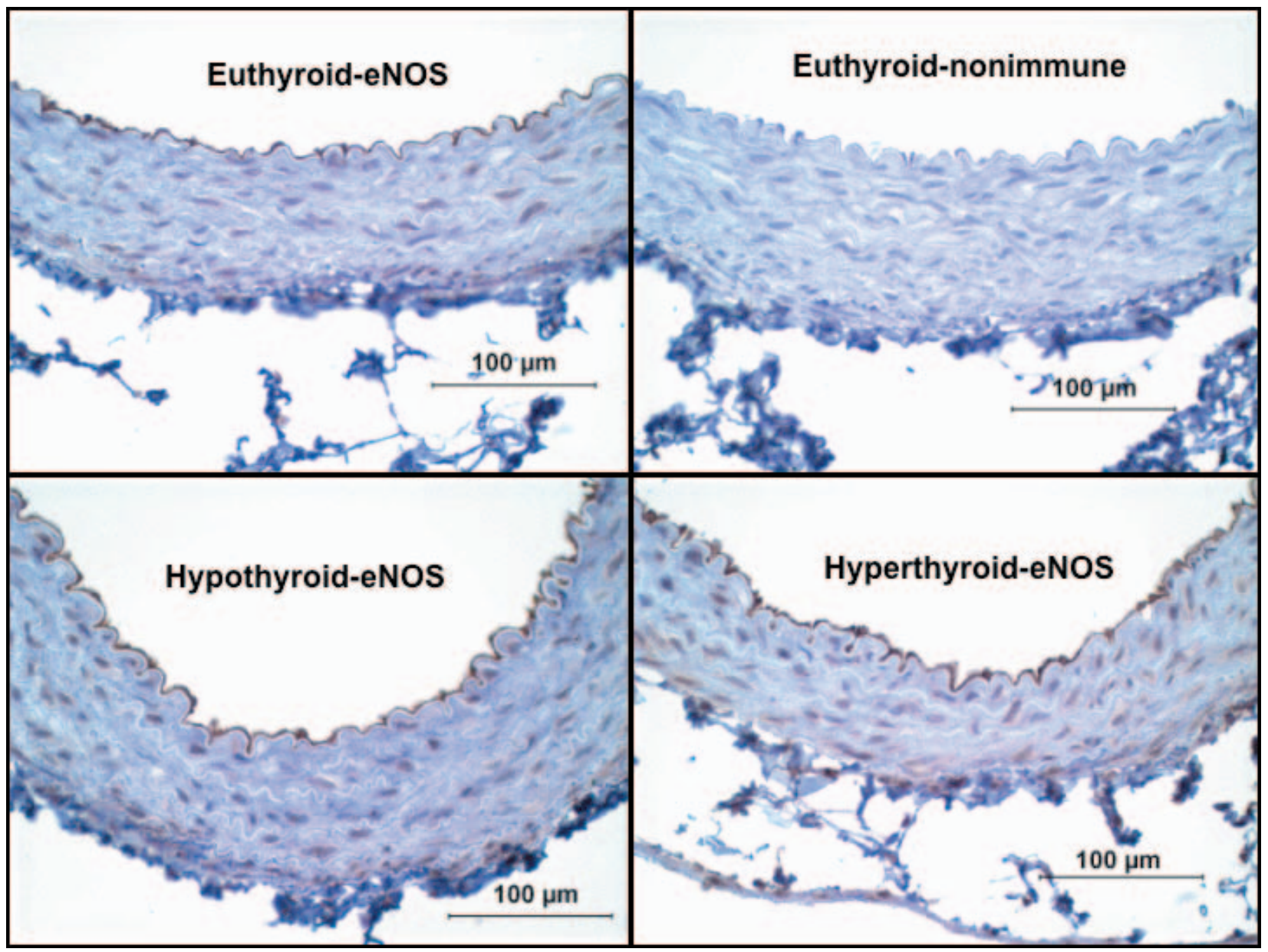

Figure 4 Immunohistochemical analysis of Euthyroid, Hypothyroid and Hyperthyroid aortae for eNOS. Vascular lumen located at the top of each panel; non-immune panel represents section not incubated with antibody to eNOS. Note positive staining for eNOS in endothelium of vascular wall.

an index of oxidative capacity, was reduced in the soleus muscle of HYPO. This effect of hypothyroidism on mitochondrial protein content of skeletal muscle has been repeatedly reported for rodent muscle by us (Delp et al. 1995, McAllister et al. 1995b, 2000) and others (e.g. Ianuzzo et al. 1977). Conversely, treatment with triiodothyronine was associated with increased citrate synthase activity, as reported previously by us (McAllister et al. 1995c, 1998, 2000) and others (e.g. Ianuzzo et al. 1977). HYPO also exhibited blunted growth; in addition, HYPER demonstrated left ventricular hypertrophy. These findings, all hallmarks of hypo- and hyperthyroidism, indicate that our rat models of thyroid disease are valid.

\section{Capacity for NO formation}

Although neither hypo- nor hyperthyroid status significantly altered NOS activity relative to the euthyroid state, the capacity for $\mathrm{NO}$ formation was clearly greater in aortae from HYPER than in those from HYPO. Our findings for aortic NOS activity in different thyroid states resemble those reported by Quesada et al. (2002). Further, these findings are consistent with earlier findings concerning vascular function. We (Delp et al. 1995, McAllister et al. 2000) and others (Vargas et al. 1995) have found endothelium-dependent dilation of rat vasculature to be blunted in hypothyroidism; conversely, we (McAllister et al. 1998, 2000) and others (Scivoletto et al. 1986, Vargas et al. 1995, Honda et al. 2000) have observed augmented endothelium-dependent vasodilation in the hyperthyroid state. Since a primary mediator of endothelium-dependent dilation in vasculature of the rat is NO (Delp et al. 1993), our findings collectively suggest that alterations in endothelium-dependent vasodilation with changes in thyroid status are, in part, accounted for by an altered capacity for NO formation. It is clear, however, that changes in NO formation do not solely account for altered endothelium-dependent vasodilation. Vascular function 


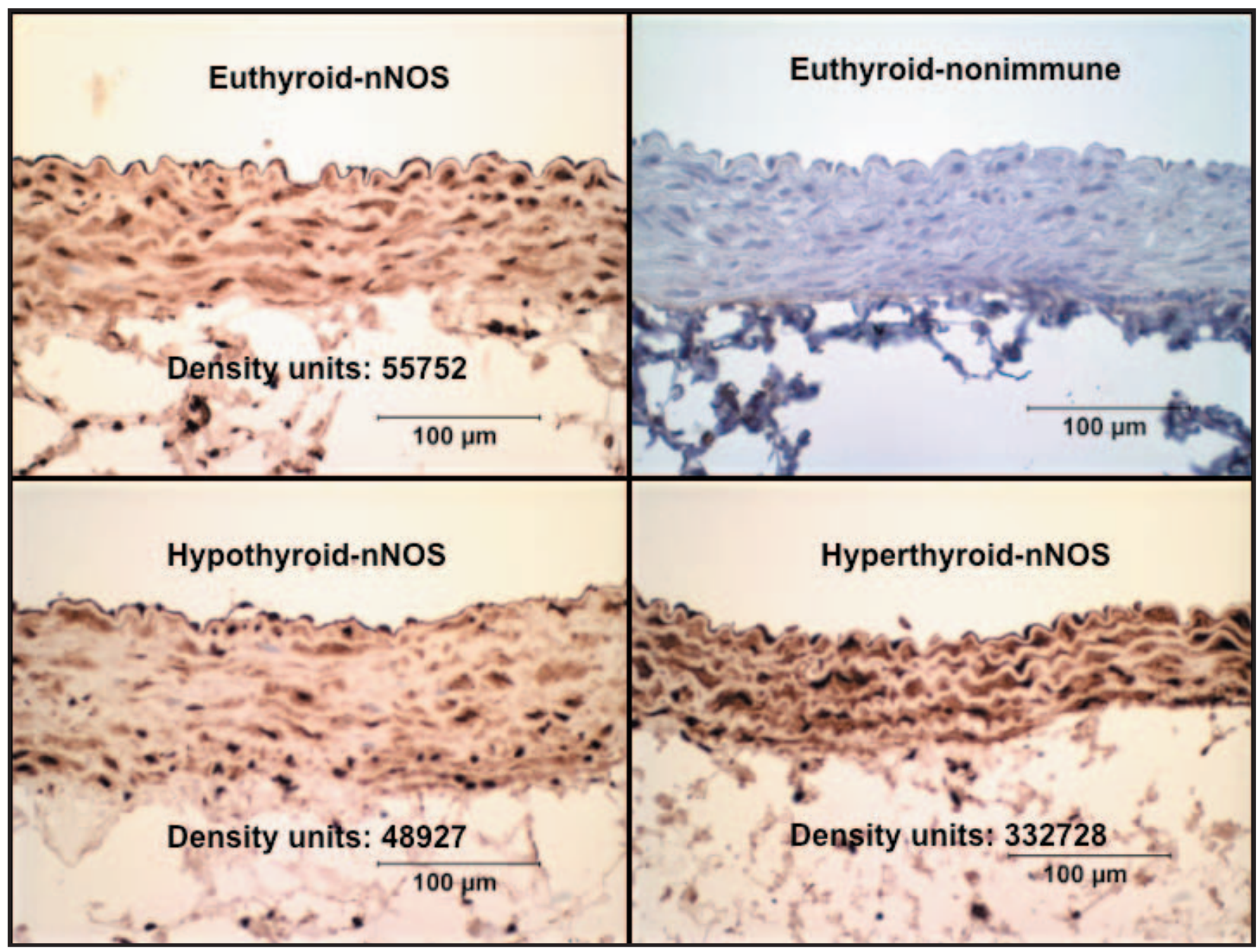

Figure 5 Immunohistochemical analysis of Euthyroid, Hypothyroid and Hyperthyroid aortae for nNOS. Vascular lumen located at top of each panel; non-immune panel represents a section not incubated with antibody to nNOS. Note positive staining for nNOS in smooth muscle of vascular wall, and greater staining for nNOS in the Hyperthyroid panel.

studies have demonstrated differences in endotheliumdependent dilation among EUT, HYPO and HYPER, whereas NOS activity in the present study only differed significantly between HYPO and HYPER. Changes in formation of vasodilatory prostaglandins and/or endothelium-derived hyperpolarizing factors may also contribute to alterations in endothelium-dependent vasodilation with altered thyroid status.

Interestingly, both eNOS and nNOS protein expression were modulated in HYPO and HYPER in a manner consistent with NOS activity. Our data for NOS activity represent capacity for $\mathrm{Ca}^{2+}$-dependent $\mathrm{NO}$ formation; eNOS and nNOS are $\mathrm{Ca}^{2+}$-dependent isoforms of this enzyme (cf. Pollock et al. 1991). While the significance of modulation of eNOS expression for endotheliumdependent vasodilation is clear, that of alterations in nNOS expression with changes in thyroid status is less clear. It has been postulated that nNOS, which appears to be expressed only in vascular smooth muscle, may modulate contractile mechanisms (Brophy et al. 2000). Our immunohistochemical findings are in agreement with this earlier study; indeed, there was a marked increase in nNOS expression in vascular smooth muscle of HYPER aorta (Fig. 5). While the functional significance of this finding is uncertain, increased nNOS expression likely contributed to greater $\mathrm{Ca}^{2+}$-dependent NOS activity in HYPER.

Modulation of eNOS and nNOS expression by thyroid status may involve several stimuli, two of which are thyroid hormone concentration and chronic level of blood flow/shear stress. Hypo- and hyperthyroidism have effects on protein expression in most tissues (Yen 2001); their effects on skeletal muscle metabolic and contractile protein expression are particularly well established (Ianuzzo et al. 1977, Caiozzo \& Haddad 1996). It is unknown whether thyroid hormone affects protein expression in the vascular wall. Effects of chronically increased blood flow/shear stress on the vascular endothelium are better understood. 

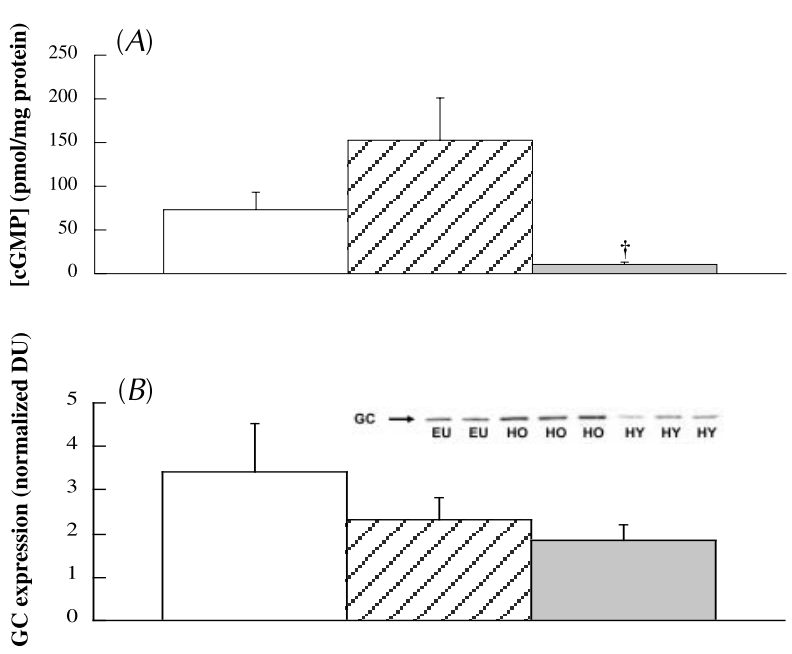

Figure 6 (A) Concentration of cGMP in aortae stimulated with sodium nitroprusside. Values are means \pm S.E.; $n=8,8$ and 8 for Euthyroid (open bar), Hypothyroid (hatched bar) and Hyperthyroid (stippled bar) respectively. †Different from Hypothyroid, $P<0 \cdot 05$. (B) Expression of GC protein. Values are mean \pm S.E.; $n=6,5$ and 8 for Euthyroid (open bar), Hypothyroid (hatched bar) and Hyperthyroid (stippled bar) respectively. DU, densitometry units, normalized to GAPDH. There are no differences in GC expression among groups. Inset is a representative Western blot for GC. EU, Euthyroid; HO, Hypothyroid; HY, Hyperthyroid.

Studies using cultured endothelial cells exposed to increased shear stress (Nishida et al. 1992, Noris et al. 1995, Ranjan et al. 1995, Uematsu et al. 1995), and whole blood vessels studied in vitro after a period of chronically increased blood flow (Miller et al. 1986, Miller \& Vanhoutte 1988, Miller \& Burnett 1992, Nadaud et al. 1996), have reported increased endothelium-dependent vasodilation, increased NOS activity, and/or increased eNOS expression. It is therefore possible that increased cardiac output, a feature of human hyperthyroidism (Martin et al. 1991) and animal models of the disease (cf. Morkin et al. 1983), led to increased eNOS expression in HYPER via a chronic increase in shear stress on the aortic endothelium.

\section{Response to NO}

An unexpected finding in this study was that thyroid status also modulated the response of vascular smooth muscle to NO. While aortae from EUT, HYPO and HYPER all demonstrated large increases in cGMP concentration upon stimulation with an $\mathrm{NO}$ donor (i.e. stimulated vs basal cGMP concentrations), the increase in HYPO was markedly greater than that in HYPER (Fig. 6A). Normalizing cGMP concentration to tissue weight, rather than tissue protein concentration, did not change this finding. We were, however, unable to confirm depressed capacity for cGMP formation in HYPER when we determined cGMP release from vascular smooth muscle. Nonetheless, our data uniformly indicate that capacity for cGMP formation is increased in HYPO. This finding was unexpected, since we have previously found that aortae from hypothyroid rats (Delp et al. 1995, McAllister et al. 2000) exhibit normal relaxation responses to sodium nitroprusside at the same concentration used in the present study to stimulate GC. Further, we found that GC protein expression was similar among EUT, HYPO and HYPER, a finding inconsistent with alterations in the capacity for stimulated cGMP formation. Differences in breakdown of cGMP do not underlie our findings, since we included isobutylmethylxanthine, a phosphodiesterase inhibitor, in our assay system to eliminate cGMP breakdown as a confounding variable. These findings emphasize the complexity of vascular cGMP formation and may indicate that acute regulation of GC (e.g. phosphorylation of GC, cytosolic $\mathrm{Ca}^{2+}$ concentration; cf. Friebe \& Koesling 2003), rather than simple modulation of GC protein amount, is most important in determining cGMP formation in different thyroid states.

\section{Perspectives}

Exercise intolerance in hypothyroidism appears to be largely accounted for by cardiovascular dysfunction. Skeletal muscle blood flow during exercise is blunted (McAllister et al. 1995b), as is endothelium-dependent vasodilation (Delp et al. 1995, Vargas et al. 1995, McAllister et al. 2000). Conversely, hyperthyroidism is associated with augmented muscle blood flow during exercise (Frey 1967, McAllister et al. 1995c) and with enhanced endothelium-dependent vasodilation (Scivoletto et al. 1986, Vargas et al. 1995, McAllister et al. 1998, 2000, Honda et al. 2000). Our findings for NOS activity, as well as for eNOS and nNOS protein expression, suggest that changes in capacity for $\mathrm{NO}$ formation contribute to alterations in endothelium-dependent vasodilation in hypo- and hyperthyroidism. They may, therefore, contribute to the mechanistic basis for altered skeletal muscle blood flow during exercise in thyroid disease states.

\section{Acknowledgements}

The technical assistance of Amy Barker, Emily Buhr, Stephanie Coovert and Kasee Hildenbrand is gratefully acknowledged. Also acknowledged is provision of equipment essential to the conduct of this research by Drs Lisa Freeman and George Stewart.

\section{Funding}

This research was supported by NIH HL 57226 and AHA-KS-98-GB-25, as well as by a Research Career Enhancement Award from the American Physiological 
Society. T K S was a Howard Hughes Medical Institute (HHMI) Research Scholar, supported by a HHMI Undergraduate Biology Education Program grant to Kansas State University. The authors declare that there is no conflict of interest that would prejudice the impartiality of this scientific work.

\section{References}

Brophy CM, Knoepp L, Xin J \& Pollock JS 2000 Functional expression of NOS 1 in vascular smooth muscle. American Journal of Physiology - Heart and Circulatory Physiology 278 H991-H997.

Caiozzo VJ \& Haddad F 1996 Thyroid hormone: modulation of muscle structure, function, and adaptive responses to mechanical loading. Exercise and Sport Sciences Reviews 24 321-361.

Canaris GJ, Manowitz NR, Mayor G \& Ridgway EC 2000 The Colorado thyroid disease prevalence study. Archives of Internal Medicine 160 526-534.

Danforth E \& Burger AG 1989 The impact of nutrition on thyroid hormone physiology and action. Annual Reviews of Nutrition 9 201-227.

Delp MD, McAllister RM \& Laughlin MH 1993 Exercise training alters endothelium-dependent vasoreactivity of rat abdominal aorta. Journal of Applied Physiology 75 1354-1363.

Delp MD, McAllister RM \& Laughlin MH 1995 Exercise training alters aortic vascular reactivity in hypothyroid rats. American Journal of Physiology - Heart and Circulatory Physiology 268 H1428-H1435.

Frey HMM 1967 Peripheral circulatory and metabolic consequences of thyrotoxicosis: IV. Blood flow in resting and working calf muscle in patients with thyrotoxicosis before and after treatment. Scandinavian Journal of Clinical and Laboratory Investigation 19 143-148.

Friebe A \& Koesling D 2003 Regulation of nitric oxide-sensitive guanylyl cyclase. Circulation Research 93 96-105.

Henderson KK, Turk JR, Rush JWE \& Laughlin MH 2004 Endothelial function in coronary arterioles from pigs with early-stage coronary disease induced by high-fat, high-cholesterol diet: effect of exercise. Journal of Applied Physiology 97 1159-1168.

Honda H, Iwata T, Mochizuki T \& Kogo H 2000 Changes in vascular reactivity induced by acute hyperthyroidism in isolated aortae. General Pharmacology 34 429-434.

Ianuzzo D, Patel P, Chen V, O’Brien P \& Williams C 1977 Thyroidal trophic influence on skeletal muscle myosin. Nature $\mathbf{2 7 0}$ 74-76.

Martin WH, Spina RJ, Korte E, Yarasheski KE, Angelopoulos TJ, Nemeth PM \& Saffitz JE 1991 Mechanisms of impaired exercise capacity in short duration experimental hyperthyroidism. Journal of Clinical Investigation 88 2047-2053.

McAllister RM, Delp MD \& Laughlin MH 1995a Thyroid status and exercise tolerance: cardiovascular and metabolic considerations. Sports Medicine 20 189-198.

McAllister RM, Delp MD, Thayer KA \& Laughlin MH 19956 Muscle blood flow during exercise in sedentary and trained hypothyroid rats. American Journal of Physiology - Heart and Circulatory Physiology 268 H1949-H1954.

McAllister RM, Sansone JC \& Laughlin MH 1995c Effects of hyperthyroidism on muscle blood flow during exercise in rats. American Journal of Physiology - Heart and Circulatory Physiology 268 H330-H335.

McAllister RM, Grossenburg VD, Delp MD \& Laughlin MH 1998 Effects of hyperthyroidism on vascular contractile and relaxation responses. American Journal of Physiology - Endocrinology and Metabolism 274 E946-E953.
McAllister RM, Luther KL \& Pfeifer PC 2000 Thyroid status and response to endothelin-1 in rat arterial vessels. American Journal of Physiology - Endocrinology and Metabolism 279 E252-E259.

Miller VM \& Burnett JC 1992 Modulation of NO and endothelin by chronic increases in blood flow in canine femoral arteries. American Journal of Physiology - Heart and Circulatory Physiology 263 H103-H108.

Miller VM \& Vanhoutte PM 1988 Enhanced release of endothelium-derived factor(s) by chronic increases in blood flow. American Journal of Physiology - Heart and Circulatory Physiology 255 $\mathrm{H} 446-\mathrm{H} 451$.

Miller VM, Aarhus LL \& Vanhoutte PM 1986 Modulation of endothelium-dependent responses by chronic alterations of blood flow. American Journal of Physiology - Heart and Circulatory Physiology 251 H520-H527.

Morkin E, Flink IL \& Goldman S 1983 Biochemical and physiologic effects of thyroid hormone on cardiac performance. Progress in Cardiovascular Disease 25 435-464.

Nadaud S, Philippe M, Arnal J-F, Michel J-B \& Soubrier F 1996 Sustained increase in aortic endothelial nitric oxide synthase expression in vivo in a model of chronic high blood flow. Circulation Research 79 857-863.

Nishida K, Harrison DG, Navas JP, Fisher AA, Dockery SP, Uematsu M, Nerem RM, Alexander RW \& Murphy TJ 1992 Molecular cloning and characterization of the constitutive bovine aortic endothelial cell nitric oxide synthase. Journal of Clinical Investigation $902092-2096$.

Noris M, Morigi M, Donadelli R, Aiello S, Foppolo M, Todeschini M, Orisio S, Remuzzi G \& Remuzzi A 1995 Nitric oxide synthesis by cultured endothelial cells is modulated by flow conditions. Circulation Research 76 536-543.

Pollock JS, Forstermann U, Mitchell JA, Warner TD, Schmidt HHHW, Nakane M \& Murad F 1991 Purification and characterization of particulate endothelium-derived relaxing factor synthase from cultured and native bovine aortic endothelial cells. PNAS 88 10480-10484.

Quesada A, Sainz J, Wangensteen R, Rodriguez-Gomez I, Vargas F \& Osuna A 2002 Nitric oxide synthase activity in hyperthyroid and hypothyroid rats. European Journal of Endocrinology 147 117-122.

Ranjan V, Xiao Z \& Diamond SL 1995 Constitutive NOS expression in cultured endothelial cells is elevated by fluid shear stress. American Journal of Physiology - Heart and Circulatory Physiology 269 $\mathrm{H} 550-\mathrm{H} 555$.

Scivoletto R, Fortes ZB \& Garcia-Leme J 1986 Thyroid hormones and vascular reactivity: role of the endothelial cell. European Journal of Pharmacology 129 271-278.

Srere PA 1969 Citrate synthase. Methods in Enzymology 13 3-5.

Steel RGD \& Torrie JH 1980 Principles and Procedures of Statistics, edn 2, pp 102-104, 137-153, 185-186. New York: McGraw-Hill.

Tunbridge WMG \& Caldwell G 1991 The epidemiology of thyroid diseases. In The Thyroid: a Fundamental and Clinical Text, edn 6, pp 578-587. Eds LE Braverman \& RD Utiger. Philadelphia, PA: JB Lippincott.

Uematsu M, Ohara Y, Navas JP, Nishida K, Murphy TJ, Alexander RW, Nerem RM \& Harrison DG 1995 Regulation of endothelial cell nitric oxide synthase mRNA expression by shear stress. American Journal of Physiology - Cell Physiology 269 C1371-C1378.

Vargas F, Fernandez-Rivas A, Estan JG \& del Rio CG 1995 Endothelium-dependent and endothelium-independent vasodilation in hyperthyroid and hypothyroid rats. Pharmacology 51 308-314.

Yen PM 2001 Physiological and molecular basis of thyroid hormone action. Physiological Reviews 81 1097-1142.

Received 9 December 2004

Accepted 6 January 2005 